\title{
Intermediate Care Units: Zwischen ICU und Bettenstation
}

\author{
Luca Lavina ${ }^{a}$, Michael Wehrlib, Thierry Fumeaux \\ ${ }^{a}$ Generalsekretariat SGI \\ ${ }^{b}$ Geschäftsführender Präsident SGI \\ c Prof. Dr. med., Präsident Ärzte SGI, Mitglied FMH
}

Patientinnen und Patienten sollen während eines stationären Aufenthalts im Spital zur richtigen Zeit am richtigen Ort kompetent behandelt werden. Wo kümmert man sich um Patienten, die für eine Normalstation zu krank, gleichzeitig aber nicht auf die aufwendigen Massnahmen der Intensivmedizin angewiesen sind? Lange gab es auf diese Frage keine eindeutige Antwort, Patienten wurden auf Abteilungen behandelt, die nicht für sie konzipiert wurden. Seit knapp zwanzig Jahren entstehen in Schweizer Spitälern jedoch Abteilungen, die sich auf die Behandlung genau dieser Patienten spezialisiert haben und die Lücke zwischen Bettenstation und Intensive Care Unit (ICU) effektiv schliessen. Seit letztem Herbst werden diese sogenannten Intermediate-Care- oder kurz IMC-Units von einer interdisziplinären und interprofessionellen Arbeitsgruppe unter der Federführung der Schweizerischen Gesellschaft für Intensivmedizin (SGI) visitiert und anerkannt. Weil die SGI von der hohen Relevanz dieser IMC-Units für das Gesundheitssystem der Schweiz überzeugt ist, hat die Fachgesellschaft sie zum Hauptthema des diesjährigen SGI Symposiums erkoren, das Anfang März im Berner Inselspital stattfand.

\section{Offiziell anerkannte IMC-Units}

Die Geschichte der Intermediate Care Unit beginnt gegen Ende der 60er Jahre. Herzinfarkt-Patienten mit einem erhöhten Komplikationsrisiko wurden auf sogenannten Coronary Care Units gruppiert und von spezialisiertem Fachpersonal umfassend betreut, wodurch die Mortalität dieser Patientengruppe markant reduziert werden konnte.

Nach dem Vorbild der Coronary Care Units begann man in der Schweiz in den 90er Jahren mit dem Aufbau von Intermediate Care Units. Doch erst ab 2011 erarbeiteten neun schweizerische Fachgesellschaften unter der Leitung der SGI spezifische Richtlinien für IMCUnits, welche die Behandlungsqualität auf diesen Abteilungen gewährleisten sollen. Seit letztem Sommer können sich IMC-Units nun offiziell anerkennen lassen und ihre Leistungen somit in eigens dafür vorgesehenen DRGs differenziert abrechnen, erwähnt Constanze Hergeth von SwissDRG.

Wie Jolanda Contartese, Leitende Ärztin der IMC-Unit am Kantonsspital Baden und Vize-Präsidentin der Kommission für die Anerkennung von Intermediate Care Units (KAIMC), betont, widmen sich die meisten IMC-Units in der Schweiz einem bestimmten Krankheitsbild, wie im Falle der Coronary Care Units, oder einer konkreten medizinischen Disziplin. Es gibt jedoch auch eine ganze Reihe von allgemeinen oder interdisziplinären IMC-Units in der Schweiz ohne fachliche Spezialisierung. IMC-Units können zudem unabhängig für sich funktionieren oder an andere Stationen - häufig an eine ICU - im selben oder sogar einem anderen Spital angeschlossen sein.

\section{Flexibilität und Vielfalt als Stärken}

Das genaue Therapieangebot und die Beziehung zu anderen Stationen und Kliniken hängen zu einem grossen Teil von der IMC, ihren Mitarbeitenden, dem Spital und den Patienten ab. Die erarbeiteten Richtlinien zur Anerkennung von IMC-Units erlauben eine gewisse Flexibilität und deshalb eine grosse Vielfalt an solchen Stationen - genau hier steckt eine ihrer grossen Stärken. Laut Marco Maggiorini, Past-Präsident der SGI, gibt man auf diese Weise auch kleineren Institutionen, für welche die Bewirtschaftung einer Intensivstation $\mathrm{zu}$ aufwendig ist, die Möglichkeit, IMC-Units einzurichten. Diese sollten dafür aber eine Kooperation mit einem Spital eingehen, das eine Intensivstation führt. Allen IMC-Units bleibt gemein, dass sie Patienten mit erhöhtem Pflegebedarf behandeln. Auf einer ICU betreut eine Pflegefachperson ein bis zwei, auf einer Normalstation bis zu acht (nachts bis zu 24) Patienten. Dazwischen liegt die IMC mit drei bis vier Patienten pro Pflegefachperson. Für diese Patienten gibt es drei Pfade, die auf eine IMC-Unit führen, so Elisabeth Allemann vom Inselspital Bern. Manche Patienten werden nach Operationen auf die IMC-Unit verlegt, an- 
dere werden notfallmässig eingeliefert, häufig auch, weil das Monitoring auf der Bettenstation nicht mehr ausreicht und verstärkt werden muss. In diesen Fällen spricht man von der IMC-Unit als Step-Up-Unit. Kommt ein Patient oder eine Patientin nach Stabilisierung der Vitalparameter von der Intensivstation auf die IMCUnit, ist diese eine Step-Down-Unit. Nach demselben Prinzip funktioniert die Verlegung von der IMC-Unit auf andere Abteilungen. Geht es einem Patienten besser, wird dieser auf eine Bettenstation verlegt. Verschlechtert sich der Gesundheitszustand, etwa weil mehr als ein Organ akut versagt, geschieht wiederum ein Step-Up von der IMC auf eine Intensivstation.

\section{Massgeschneiderte Versorgung}

Intermediate Care Units können also vielen unterschiedlichen Patienten mit ebenso vielen Krankheitsbildern und -geschichten eine massgeschneiderte medizinische Versorgung bieten und somit aus einem zweistufigen Behandlungskonzept in einem Spital ein dreistufiges machen. Das steigert den Nutzungsgrad der Intensivstationen und Allgemeinstationen, weil diese sich um jene Patienten kümmern können, auf deren Behandlung sie tatsächlich ausgelegt sind.

Wie auf Intensivstationen ist die Arbeit auf Intermediate Care Units stark von Interprofessionalität und Interdisziplinarität geprägt. Ärzte, Ärztinnen und Pflegende, aber auch Therapeuten unterschiedlicher Disziplinen arbeiten hier eng zusammen - was laut Martine Louis Simonet aus dem HUG in Genf neues Wissen generiert und so letztlich dem Patienten zugutekommt. Beide Berufsgruppen sind der Meinung, dass eine Weiterbildung auf dem Gebiet der Intermediate Care sowohl für Pflegende und Ärzte notwendig ist: Für Thierry Fumeaux, Präsident Ärzte der SGI, ist eine Spezialisierung der medizinischen Belegschaft einer IMC in Zukunft durchaus vorstellbar. Auch für Hans Richter, Berufsbildungsverantwortlicher NDS HF Intensivpflege, ist klar, dass IMC-Pflege spezifische Kompetenzen erfordert und gerade eine praktische Weiterbildung in diesem Bereich von grosser Bedeutung ist. Zurzeit werden auf nationaler Ebene von OdASanté minimale Anforderungen für eine vereinheitlichte IMCWeiterbildung von diplomierten Pflegefachpersonen erarbeitet.

Wie im Behandlungsteam auf der IMC-Unit ist auch in der KAIMC Interprofessionalität und Interdisziplinarität von grösster Bedeutung. Die Kommission besteht aus mittlerweile mehr als 30 engagierten Vertreterinnen und Vertretern (Ärzteschaft und Pflege) aus der Intensivmedizin, der Anästhesiologie und Reanimation, der Kardiologie, der Chirurgie, der Kinderchirurgie, der Allgemeinen Inneren Medizin, der Pädiatrie, der klinischen Neurowissenschaften und der Neonatologie. Ohne die interprofessionelle und interdisziplinäre Zusammenarbeit dieser Kommissionsmitglieder wäre die Anerkennung von IMC-Units schlicht nicht durchführbar.

Trotz grundsätzlicher Zustimmung zu einer solchen Schnittstellenabteilung und den erarbeiteten Qualitätskriterien wurden am Symposium auch Anregungen für eine potentielle Revision der Richtlinien geäussert. Während Peter Altherr, Leiter des Amts für Gesundheitsversorgung des Kantons St. Gallen, sich eine bessere Verknüpfung der IMC-Richtlinien mit kantonalen Spitalplanungsanforderungen wünscht, bestehen die

\section{Wie auf Intensivstationen ist die Arbeit auf} Intermediate Care Units stark von Interprofessionalität und Interdisziplinarität geprägt.

Richtlinien für Jürg Hodler, den ärztlichen Direktor des USZ, aus zu vielen Bauvorschriften. Medizinische Vorgaben kämen auf den zwanzig Seiten deshalb zu kurz. Bei vielen baulichen Kriterien, so Jolanda Contartese, handle es sich um sogenannte S2-Kriterien, von denen für eine erfolgreiche Anerkennung nicht alle, sondern nur 60 Prozent erfüllt werden müssen und die genau deshalb für eine gewisse Flexibilität der Qualitätskriterien sorgen. "Wir möchten vielen IMC-Units in der Schweiz die Chance geben, sich anerkennen zu lassen, weil es unser primäres Anliegen ist, die Behandlungsqualität der Intermediate Care zu steigern und Mitarbeiter so in der Folge auch die Möglichkeit haben, sich in diesem Gebiet weiterzubilden", so Jolanda Contartese.

Am grossen Interesse an der Anerkennung von IMCUnits scheinen diese Anregungen nichts zu ändern: Per Ende April 2017 wurden bereits 22 IMC-Units von der IMC-Anerkennungskommission anerkannt, neun weitere Abteilungen haben sich für eine Anerkennung angemeldet - Tendenz steigend.

Alle Beiträge des SGI Symposiums 2017 können Sie sich als Webcasting unter www.sgi-ssmi.ch nochmals anschauen. Weitere Informationen zu Intermediate Care finden Sie unter www.swiss-imc.ch 